\title{
Qualitative analysis of electron states in nanoobjects
}

\author{
B.A. Lukiyanets \\ National University “Lviv Polytechnic”, 12, Bandera str., 79013 Lviv, Ukraine \\ E-mail: lukbog@i.ua; lukbogster@gmail.com
}

\begin{abstract}
Tendencies of change of the electron energy states at transition from a "rigid" nanoobject to a nanoobject, in which taken into account is the influence of medium on it, are considered. Proposed is the model describing the system of nanoparticles, i.e. nanocluster. Position of the ground state depending on both the size of nanoparticle and that of nanocluster is analyzed.
\end{abstract}

Keywords: nanoobject, nanocluster, electron state.

Manuscript received 19.12.06; accepted for publication 26.03.07; published online 01.06.07.

\section{Introduction}

The energy spectrum of an electron is a basis to understand physics of small particles or nanoobjects. There are many methods to simulate behaviour of nanoobjects. One of them connects peculiarities of the nanoobjects with surface effects. It is obvious that the surface effects in the nanoobjects cannot be ignored as in the case of bulk crystals: the specific weight of the surface atoms in nanoobjects is higher than in macroobjects.

Another method was proposed in [1], where in description of small particles the attention was focused on their discrete structure. Author postulated electron states with equidistant levels. In the framework of the approximation, the calculated electron heat capacity has an exponential decrease with temperature, while in macroobjects this decrease is linear.

Sometimes the electron states in nanoobjects are analyzed on the basis of band theory. But it may be considered as a zero approximation since nanoobjects are no ideal crystals. On the other hand, there are inapplicable results of the quantum theory of an atom. Nanoobjects are something intermediate between atoms and a crystal with some probably nontrivial distinctions both from an atom and a crystal. Ascertaining these distinctions with the purpose of their possible practical use or forecasting behavior of the nanoobjects when changing their parameters is an actual problem of scientific investigations.

\section{Model and a calculation scheme}

Current state of theoretical investigations of nanoobjects justifies using simplified models to understand physical effects in them. As an example one can give consideration of one-dimensional quantum-mechanical problem to description of a resonance transition in the model of a nanoobject with the squared potential wells $[2,3]$.

Let us analyze the electron spectrum of a nanoobject on a basis of the one-dimensional model that allows modifying the size of the nanoobject, its energy parameters, namely: time-independent Schrödinger equation with potentials presented in Fig. 1. The analytical expression for the potentials has the following form

$$
\begin{aligned}
& U(x)=\left\{\begin{array}{c}
U_{0} \\
0 \Rightarrow\left\{\begin{array}{c}
U_{0} \\
\text { const } \cdot x^{2} \Rightarrow \\
U_{0}
\end{array}\right. \\
a
\end{array}\right\} \\
& \Rightarrow\left\{\begin{array}{c}
U_{0}, x \in(-\infty,-a] \\
\operatorname{const} \cdot x^{2}+A_{0} \cos (p x), x \in[-a, a] \\
U_{0} x \in[a, \infty)
\end{array}\right.
\end{aligned}
$$

The difference between potentials $1 a$ and $1 b$ lies in their analytical forms within the spatial region $[-a, a]$ that is the region of the nanoobject. These potentials may be considered with some logical order as approximation to description of a nanoobject. Potential $1 a$ designs a "rigid" nanoobject of size $2 a$ without any its distortion by a medium. In one's part potential $1 b$ designs more real object with edge distortion caused by a medium. Since in both cases sizes of the nanoobjects (2a) and potential of a medium $\left(U_{0}\right)$ are the same, then const in $U(x)$ is equal $\frac{U_{0}}{a^{2}}$. 

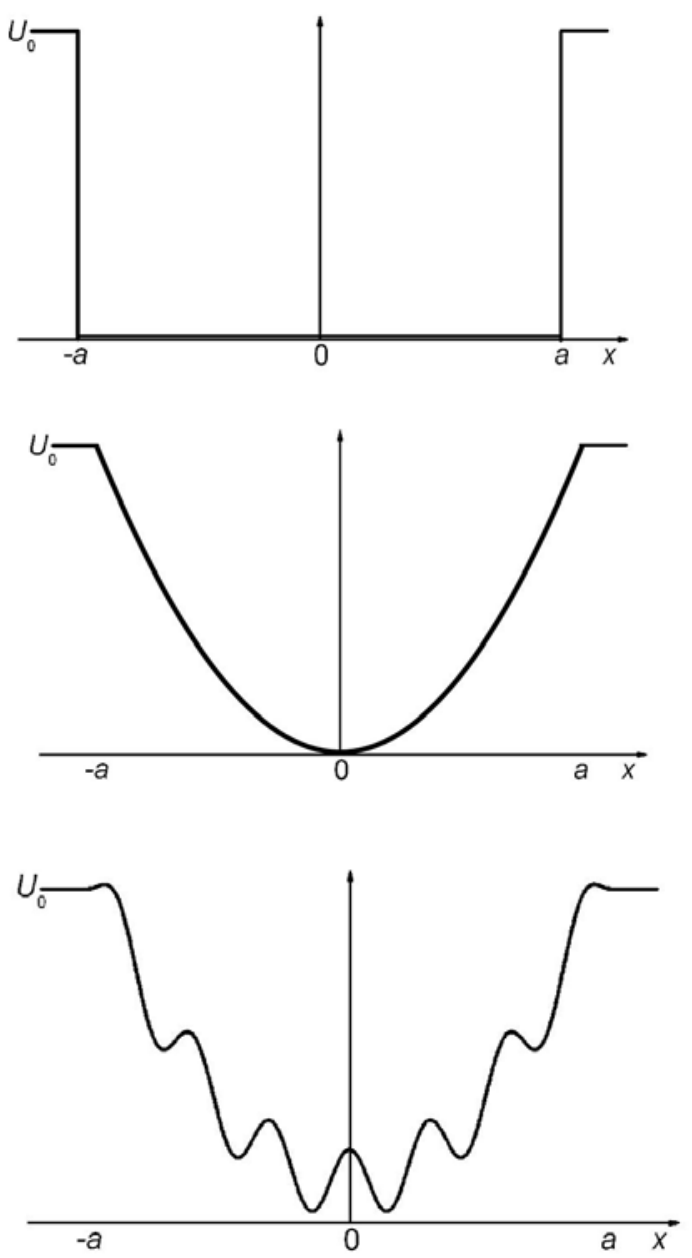

Fig. 1. Potentials that design a nanoobject.

The potential $1 c$ is the same potential $1 b$ supplemented with a periodical function that allows to design a discrete structure of the nanoobject or the system of the nanoparticles, i.e. a nanocluster.

Let us analyze a qualitative change of an electron spectrum at a successive transition from the potential $1 a$ to potential $1 b$.

\section{The case of the potential $1 a$}

It is known (see, e.g. [4]) that the solution of the timeindependent Schrödinger equation

$\left\{-\frac{\hbar^{2}}{2 m} \frac{d^{2}}{d x^{2}}+U(x)\right\} \psi(x)=E \psi(x)$

needs its finding (taking into account non-analyticity of the potential) in three regions $(-\infty,-a],[-a, a],[a, \infty)$. In this case, in the region $x>a$ the wave function can be written as

$\psi(x)=A \exp (-\gamma x)$ with $\gamma^{2}=\frac{2 m}{\hbar^{2}}\left(U_{0}-E\right)$, and in the region $x \in[-a, a]$ there are two types of the solution - solutions with even and odd parity:

$\psi(x)=$ const $\cos \frac{\pi n}{2 a} x$, for odd $n$

and

$\psi(x)=$ const $\cdot \sin \frac{\pi n}{2 a} x$, for even $n$

with eigen values that are the solutions of the transcendental equation

$2 k a=n \pi-2 \arcsin \frac{\hbar k}{\sqrt{2 m U_{0}}}$,

where $k^{2}=\frac{2 m E}{\hbar^{2}}$.

Let us consider the solution of Eq. (6) in the case of the size of the nanoobject $2 a=10 \mathrm{~nm}, U_{0}=10 \mathrm{eV}$. In Fig. 2 shown are some of the lowest levels as solutions of Eq. (6). For comparison, there presented is the spectrum of an analogous problem with $U_{0} \rightarrow \infty$. It follows from it that the ground state in the problem with finite $U_{0}$ has a higher value that in the case of the infinite potential well. The second conclusion is that in the region $E<U_{0}$ the number of levels in the case of finite $U_{0}$ is larger in the interval $\left[0, U_{0}\right]$ in comparison with its number in the case of an infinite potential well.

\section{The case of the potential $1 b$}

In the region $x>a$, the wave function has the form (3), and in the region $x \in[-a, a]$ the time-independent Schrödinger equation is the problem of the quantum harmonic oscillator (see [4]). Its wave functions are

$\psi(x)=N \exp \left(-\frac{\alpha x^{2}}{2}\right) H_{n}(\sqrt{\alpha} x)$,

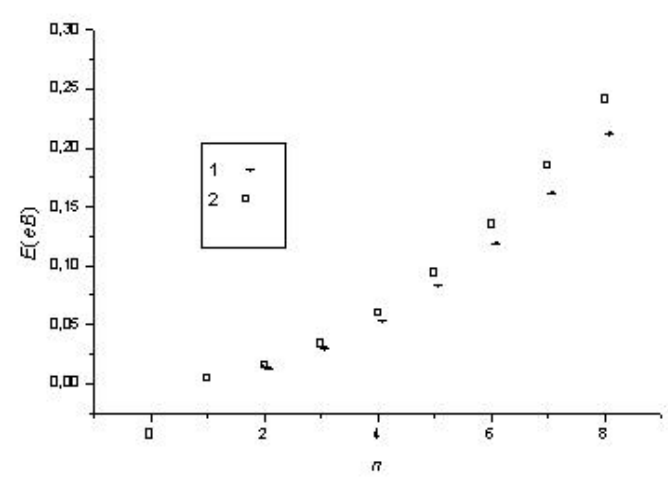

Fig. 2. Low energy levels in the case of a rectangular potential well. 
where $N$ is a normalization factor, $\alpha=\sqrt{\frac{2 m U_{0}}{\hbar^{2} a^{2}}}$, and

$H_{n}(z)=(-1)^{n} \exp \left(z^{2}\right) \frac{d^{n}}{d z^{n}} \exp \left(-z^{2}\right)(n=0,1,2, \ldots)$

is the Hermite polynomial.

Using the wave functions in the point $x=a$ gives the equation

$$
N \exp \left(-\frac{\alpha a^{2}}{2}\right) H_{n}(\sqrt{\alpha} a)=A \exp (-\gamma a),
$$

and a requirement of smoothness of the wave function in this point adds one equation more

$$
\begin{aligned}
& -\alpha a N \exp \left(-\frac{\alpha a^{2}}{2}\right) H_{n}(\sqrt{\alpha} a)+ \\
& +\left.N \exp \left(-\frac{\alpha a^{2}}{2}\right) H_{n}^{\prime}(\sqrt{\alpha} x)\right|_{x=a}=-\gamma A \exp (-\gamma a)
\end{aligned}
$$

where $H_{n}^{\prime}(\sqrt{\alpha} x)$ is the coordinate derivative of the Hermite polynomial. Using relation between the Hermite polynomial and its derivative [5]

$$
\frac{d^{k} H_{n}(z)}{d z^{k}}=n(n-1) \ldots(n-k+1) H_{n-k}(z),
$$

$0<k \leq n$,

we obtain

$$
H_{n}^{\prime}(\sqrt{\alpha} x)=\sqrt{\alpha} n H_{n-1}(\sqrt{\alpha} x) \text {. }
$$

A non-trivial solution of the system of equations (9), (10) is possible at the condition of equality to zero of the determinant, matrix elements of which are coefficients at $A$ and $N$. Dividing left and right parts of (10) by proper parts of (9) and taking into account definitions $\alpha$ and $\gamma$, we obtain

$$
1-\sqrt{\frac{U_{0}-E}{U_{0}}}-\frac{n H_{n-1}(\sqrt{\alpha} a)}{\sqrt{\alpha} a H_{n}(\sqrt{\alpha} a)}=0 .
$$

Solutions of this equation for some of the lowest states at the same parameters as before are shown in Fig. 3. In this figure, curve 1 is shown for comparison. It represents energy states of a harmonic oscillator given in the whole space, i.e. $x \in(-\infty, \infty)$. Transfer from this oscillator to the "hybrid" potential $1 b$ is accompanied, firstly, by an essentially increased value of the ground state (and states with the same $n$ will be higher, too) and, secondly, equidistance in the distribution of energy levels is lost.

The only difference between the potentials $1 a$ and $1 b$ lies in their forms within the range $[-a, a]$. As it follows from the analysis of curves in Figs 2 and 3, at transition from the potential $1 a$ to $1 b$ values of levels increase by an order of magnitude.

\section{The case of the potential $1 \mathrm{c}$}

In the framework of the perturbation theory assuming that $W_{0} \gg A_{0} \quad\left(A_{0}\right.$ is the amplitude of the harmonic term of the potential $1 c), W_{0}$ is the position of the well bottom) let us analyze the tendency of changing the ground state position obtained before. (Knowledge of this tendency depending on geometry of the nanoobject, on the form its potential is important in particular for understanding the resonance transition [2]). In Fig. 4, this level at $W_{0} \neq 0$ is one of the solutions of the equation

$1-\sqrt{\frac{U_{0}-E}{U_{0}-W_{0}}}-\frac{n H_{n-1}\left(\sqrt{\alpha_{0}} a\right)}{\sqrt{\alpha_{0}} a H_{n}\left(\sqrt{\alpha_{0}} a\right)}=0$,

where $\alpha_{0}=\sqrt{\frac{2 m\left(U_{0}-W_{0}\right)}{\hbar^{2} a^{2}}}$.

Since $H_{0}(x)=1$ and $H_{1}(x)=x$, the latter equation takes the form

$1-\sqrt{\frac{U_{0}-E}{U_{0}-W_{0}}}=\frac{1}{\sqrt{\alpha_{0}} a}$.

At $W_{0}=2 \mathrm{eV}$, its solution $E(1)=2.216 \mathrm{eV}$. Let us estimate the first correction according to the perturbation theory to this level, i.e.

$$
\langle 1|\mathrm{H}| 1\rangle=2 \int_{0}^{a} N^{2} \exp \left(-\alpha_{0} x^{2}\right) H_{1}^{2}\left(\sqrt{\alpha_{0}} x\right) A_{0} \cos (p x) d x,
$$

where $\mathrm{H}=A_{0} \cos (p x)$ is the Hamiltonian of the perturbation.

From the beginning let us determine normalization $N$. With this aim, we add the equation (9) with two unknowns $N$ and $A$ normalization requirement

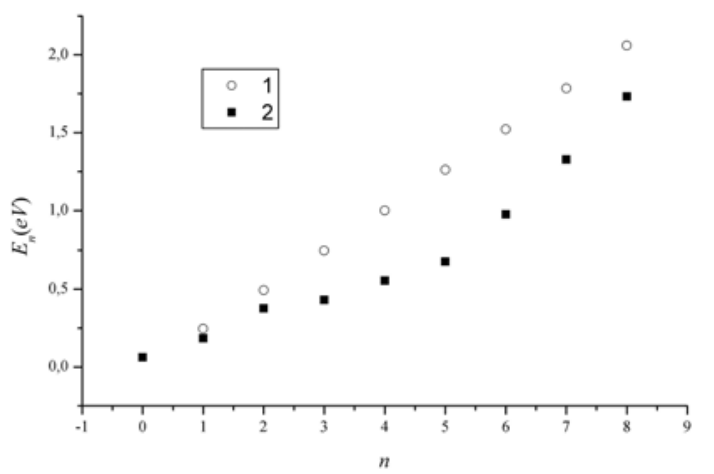

Fig. 3. Low energy levels in the case of harmonic oscillator (1), potential $1 b(2)$. 


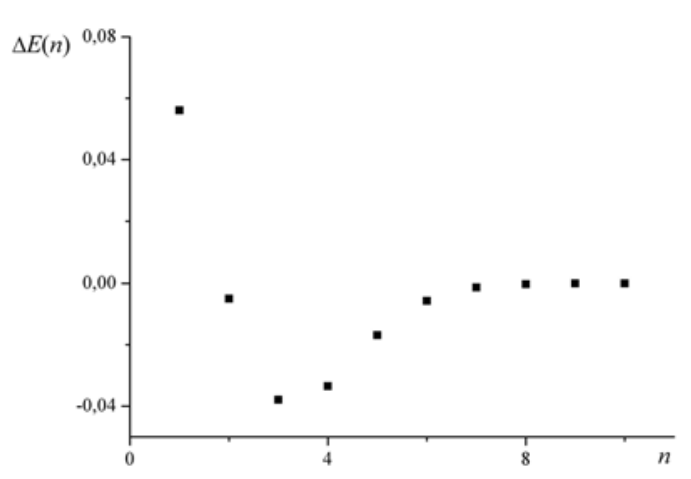

Fig. 4. Dependence of the ground state position on the size of nanoparticles.

$2 N^{2} \int_{0}^{a} \exp \left(-\alpha_{0} x^{2}\right) \alpha_{0} x^{2} d x+$

$+2 A^{2} \int_{a}^{\infty} \exp (-2 \gamma x) d x=1$

The solution of the obtained system of equations takes the form

$$
N=\frac{1}{\sqrt{2} \sqrt{i+\frac{\alpha_{0} a^{2}}{\gamma} \exp \left(-\alpha_{0} a^{2}\right)}}
$$

with

$i=\int_{0}^{a} \alpha_{0} a^{2} \exp \left(-\alpha_{0} x^{2}\right) d x$.

To calculate the integral (19), we introduce into the exponent of the integrand a parameter $S$ that in the final expressions will be equal to unity. Then

$$
\begin{aligned}
& i=-\frac{\partial}{\partial s} \int_{0}^{a} \exp \left(-s \alpha_{0} x^{2}\right) d x \Rightarrow \\
& \Rightarrow-\frac{\partial}{\partial s}\left\{\frac{1}{\sqrt{s \alpha_{0}}} \int_{0}^{a} \exp \left(-s \alpha_{0} x^{2}\right) d\left(\sqrt{s \alpha_{0}} x\right)\right\} \\
& \frac{1}{2 \sqrt{\alpha_{0}}} \operatorname{Erf}\left(\sqrt{\alpha_{0}} a\right)-\frac{a}{2} \operatorname{Erf}^{\prime}\left(\sqrt{\alpha_{0}} a\right) .
\end{aligned}
$$

Here

$$
\operatorname{Erf}(b)=\int_{0}^{b} \exp \left(-x^{2}\right) d x
$$

is an error function, and $\operatorname{Erf}^{\prime}(b)$ is its derivative with respect to the parameter $s$.

When calculating $\operatorname{Erf}(b)$, it was used its presentation by rational functions [6], namely

$$
\begin{aligned}
& \operatorname{Erf}(x)=1-\left(c_{1} t+c_{2} t^{2}+c_{3} t^{3}+c_{4} t^{4}+c_{5} t^{5}\right) \times \\
& \times \exp \left(-x^{2}\right)+\varepsilon(x)
\end{aligned}
$$

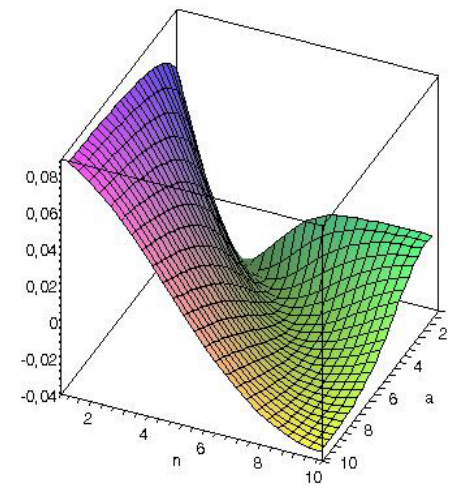

Fig. 5. Shift of the ground state position $\Delta E$ (in arbitrary units) as a function of a number of nanoparticles and their sizes.

where

$$
\begin{aligned}
& c_{1}=0.25482592, \quad c_{2}=-0.284496736, \\
& c_{3}=1.42141341, \quad c_{4}=-1.453152027, \\
& c_{5}=1.061405429, \quad t=\frac{1}{2+p_{0} x} p_{0}=0.325911, \\
& |\varepsilon(x)| \leq 1.5 \cdot 10^{-5} x \in[0, \infty) .
\end{aligned}
$$

In Fig. 4, shown is the shift of the position of the ground state $E_{0}$ depending on change of periodicity of the cosine function (with changing $n$ ) in the case of the nanocluster size as before. If minima of the cosine curve identify with positions of individual nanoparticles in the range width $2 a$, then the parameter $n$ determines a number of such nanoparticles and at the fixed size of the nanocluster $n$ determines size of the nanoparticles.

In Fig. 5, shown is a more common case, namely, a case that includes various sizes of the nanocluster from 10 to $100 \mathrm{~nm}$, at different sizes of the nanoparticles.

\section{Conclusions}

Investigations of genesis of the stationary electron states in the nanoobject from simulation of it by using a onedimensional squared potential well (model of a "rigid" nanoobject; case a) up to the potential that takes into account the surface distortion (case b) and a discrete structure or system of nanoparticles (case c) at a fixed energy depth of the well and its geometry show

- essential shift up to higher values the electron energy levels at transition from the squared potential model to the parabolic one;

- $\quad$ position of the ground state $E_{0}$ of the system of nanoparticles, i.e. a nanocluster, as a function of their sizes has non-monotonic character;

position of the ground state minimum $E_{0}$ and its value as a function of the number of nanoparticles depends on the size of the nanocluster. At the same sizes of the nanoparticles, this minimum $E_{0}$ are reached at larger sizes of the nanocluster and at those when this level falls. 


\section{References}

1. H. Frohlich // Physica 6, p. 106 (1937).

2. A.V. Tsukanov, L.B. Openov // Fizika i tekhnika poluprovodnikov 38, p. 94-101 (2004) (in Russian).

3. E.I. Golant, A.B. Pashkovskiy // Ibid. 36, p. 330337 (2002) (in Russian).
4. A.S. Davydov // Quantum Mechanics. Pergamon Press, Oxford, 1976.

5. Zh. Kampe de Feriet, P. Campbell, G. Petio, T. Fogel // Functions of Mathematical Physics. Fizmatgiz, Moscow, 1963 (in Russian).

6. Handbook of Mathematical Functions (ed. by M. Abramowitz and A. Stegun), National Bureau of Standards (1964). 